\title{
Räumliche Spezialisierung und Konzentration
}

\section{Einleitung}

Am Anfang vieler geographischer Arbeiten, vor allem aus den Bereichen der Kultur- und Wirtschaftsgeographie, steht eine detaillierte Bestandesaufnahme der wichtigsten Geofaktoren des Untersuchungsgebietes. Das in der Bestandesaufnahme ermittelte Material versucht man dann häufig mit Hilfe regionaler Kennziffern zu verdichten.

Nach MÜLLER ${ }^{1)}$ lassen sich diese regionalen Kennziffern in zwei Gruppen einteilen:

Die erste Gruppe umfaßt Kennziffern, für deren Berechnung nur Daten des jeweiligen Untersuchungsgebietes verwendet werden, wie beispielsweise «Bevölkerungsdichte», «Industriebesatz» u. ä. Diese Kennziffern werden dann oft mit denen anderer Regionen verglichen. Häufig ist der Aussagewert regionaler Kennziffern erst dann gegeben, wenn sie denjenigen anderer Regionen oder eines die Region umschließenden Gesamtgebietes gegenübergestellt werden.

Zur zweiten Gruppe zählen diejenigen Kennziffern, bei welchen man die entsprechenden Werte übergeordneter Raumeinheiten, vor allem jene des Gesamtstaates, als Vergleichsmaßstab heranzieht. Mit Hilfe derartiger Kennziffern lassen sich über die Entwicklung und Struktur eines Teilgebietes, im Vergleich zum Gesamtgebiet, recht instruktive Aussagen machen. "Um nicht der Gefahr zu erliegen, Werte des übergeordneten Raumes ungeprüft als Normwerte anzusehen - eine regionale Wirtschaft stellt nicht unbedingt ein verkleinertes Abbild der Gesamtwirtschaft dar -, können die Vergleichswerte nur dazu verwendet werden, um den Grad der strukturellen Einseitigkeit einer Region gegenüber der Vergleichsregion stärker hervortreten zu lassen2).» Solche Strukturkennziffern können nicht nur für Querschnittvergleiche benützt werden, sondern sie dienen auch als Längsschnittanalysen, d.h. für Strukturvergleiche $\mathrm{zu}$ verschiedenen Zeitpunkten. Als Beispiele für solche Kennziffern seien genannt ${ }^{3)}$ :

- Regionalfaktor (aufgespalten in Standort-und Strukturfaktor)

- Standortquotient $=$ Lokalisationsquotient $=$ Lokalisationsfaktor

- Spezialisierungskoeffizient $=$ Spezialisationskoeffizient $=$ Diversifikationskoeffizient $=$ Mischungskoeffizient
- Konzentrationskoeffizient $=$ Lokalisierungskoeffizient $=$ Lokalisationskoeffizient

- Koeffizient der räumlichen Verflechtung

Leider haben sich bis heute in der Regionalforschung noch keine einheitlichen Bezeichnungen für diese verschiedenen Strukturkennziffern durchgesetzt. Im folgenden werden für diese Kennziffern die deutschen und nicht die englisch-amerikanischen Ausdrücke verwendet.

«Diese teilräumlichen Strukturkennziffern weisen, da sie nur geringe Anforderungen an das Datenmaterial stellen, erhebliche praktische Vorteile auf. Gerade deswegen ist die Gefahr einer Überschätzung ihrer Aussagefähigkeit nicht von der Hand zu wei$\operatorname{sen}^{4}$ ).» Bei der Verwendung solcher Analysemethoden darf nie übersehen werden, daß es dabei um die statistische Darstellung regionaler Besonderheiten geht, daß aber solchen Indikatoren i. a. kein Erklärungswert beigemessen werden darf. All diese Ziffern können die Diagnose eines Gebietes erleichtern helfen, sie ersetzen aber in keinem Falle die eigentlichen Erklärungsaufgaben ${ }^{5)}$. Bei den Strukturkennziffern handelt es sich um deskriptive Techniken. Der explikative, d. h. diagnostische oder prognostische Einsatz kann nur im Zusammenhang mit allgemeinen Hypothesen oder Theorien erfolgen. Die Kennziffern können keine Erklärung bieten. Ebenfalls können die Strukturkennziffern keine Normenbegründung liefern. Eine Norm kann nur unter Berufung auf politische Zielsetzungen und niemals unmittelbar aus einer Zustandsbeschreibung bestimmt werden ${ }^{6}$. Eine Schwäche dieser Kennziffern besteht darin, daß sie sich wohl für ex-post Analysen eignen, $\mathrm{da} ß$ sie aber nur bedingt für ex-ante $Z$ wecke geeignet sind. Dazu muß allerdings betont werden, daß irgendwelche Aussagen über die Zukunft nicht ohne Kenntnisse der zurückliegenden Entwicklungen und der gegenwärtigen Situation auskommen. Eine weitere Beschränktheit der Aussagekraft dieser Indikatoren muß ebenfalls erwähnt werden: Ihre Größe hängt sehr stark davon ab, welche (willkürlichen) Einteilungen wir vornehmen, einerseits beim Untersuchungsgebiet, anderseits bei der Disaggregation des Materiales, welches wir untersuchen ${ }^{7}$. Gerade

PD Dr. Hans Elsasser, ETH Zürich, Inst. für Orts-, Regional-und Landesplanung, Weinbergstraße 35, 8006 Zürich 
bei kleineren Regionen besteht immer wieder die große Gefahr, daß Zufallsabweichungen zu Fehlinterpretationen führen können. Erst die genauen Kenntnisse über das untersuchte Gebiet erlauben dann, die wichtigsten Ursachen starker Abweichungen bei solchen Kennziffern zu erklären.

Trotz all diesen Einwänden bilden aber diese Kennziffern eine wertvolle Hilfe für die Analyse und zum Teil auch für die Diagnose eines Gebietes. Es ist deshalb m. E. erstaunlich, daß diese Indikatoren im Rahmen geographischer Arbeiten in der Schweiz praktisch nie verwendet werden ${ }^{8}$. Um nun diese Kennziffern etwas bekannter zu machen, sollen zwei davon näher erläutert und an einem schweizerischen Beispiel dargestellt werden. Es handelt sich dabei um den Spezialisierungskoeffizienten und den Konzentrationskoeffizienten. Als statistische Grundlagen werden die Daten der Eidgenössischen Industriestatistiken vom September 1967 und September 1972 verwendet, oder anders ausgedrückt, unser Untersuchungsobjekt sind die Industriebeschäftigten gemäß Industriestatistik 1967 und 1972, gegliedert nach den neunzehn industriellen Wirtschaftsgruppen 20-38) und den fünfundzwanzig Kantonen ${ }^{9}$. Um nicht nur die Querschnittanalyse zu zeigen, wurden zwei Zeitpunkte (Längsschnittanalyse) gewählt. Anstelle der Beschäftigten könnte man theoretisch, $d$. h. sofern die entsprechenden statistischen Unterlagen zurVerfügung stehen würden, auch ein anderes Industriemerkmal wählen.

Dieses Beispiel wurde aus folgendem Grunde gewählt: Eine der wichtigsten Aufgaben der Raumplanung in der Schweiz ist, einen Ausgleich zwischen wirtschaftlich starken und wirtschaftlich schwachen Gebieten anzustreben ${ }^{10}$ ). Dieses Ziel hofft man durch eine gegenüber dem Ist-Zustand ausgeglichenere regionale Verteilung von Bevölkerung und Wirtschaft (Betriebe und Beschäftigte) zu erreichen. Ausgleich bedeutet aber nicht nur, daß die regionalen Unterschiede in der Bevölkerungsdichte oder im Industriebesatz verkleinert werden sollen, sondern auch daß die Branchenstreuung in den einzelnen Regionen nicht allzu einseitig sein soll, lautet doch der zweite Grundsatz, der Arbeitsgruppe des Bundes für die Raumplanung: «In allen Landesteilen sind die räumlichen Voraussetzungen zu schaffen für eine gesunde, wettbewerbsfähige, in der Regel nicht einseitige Wirtschaftsstruktur» ${ }^{11)}$. «Eine be- stimmte Wachstumsrate ist günstiger zu bewerten, wenn sie sich auf mehrere Industriezweige verteilt, als wenn eine einseitige Abhängigkeit von einer Branche besteht, die zwar im Moment und für die nächste Zukunft hohe Wachstumsraten bringen mag, irgendwann aber auch eine um so größere Krise heraufbeschwören kann ${ }^{12}$ ).» Dazu schreibt BICKEL: «Die starke Spezialisierung eines Gebietes auf eine ganz bestimmte Industrie birgt stets große Gefahren in sich. Zwar erstrecken sich konjunkturelle Schwankungen der Wirtschaft in der Regel auf alle Industrien: in der Depression geht es allen schlecht, im Aufschwung allen gut. Aber es geht doch nicht allen gleich schlecht und nicht allen gleich gut. Die Mischung der Wirtschaftszweige gleicht die konjunkturellen Schwankungen in einem bestimmten Gebiet etwas aus. Vor allem aber besteht stets die Gefahr struktureller Krisen, die dann ein monoindustrielles Gebiet hart treffen können ${ }^{13}$ ).» Wie weit eine regionale Streuung der Industriebranchen gehen soll, bzw. ob eine Region «mäßig aber regelmäßig» wachsen oder ob sie stattdessen überdurchschnittliche Wachstumsaussichten mit einer einseitigen Branchenstruktur und damit höherem Risiko erkaufen soll, die Antwort auf diese Fragen zu finden, ist nicht Aufgabe des Wissenschafters, sondern des Politikers. Die Wissenschaft kann aber dazu Entscheidungsunterlagen liefern, indem sie Alternativen und Konsequenzen aufzeigt. Die Annäherung der regionalen Branchenstruktur an die nationale Branchenstruktur stellt keineswegs schon das anzustrebende Optimum dar, vielmehr ist der nationale Durchschnitt hierbei nur die Basis der Relativierung, die jederzeit durch eine von der politischen Zielsetzung vorgegebene Bezugsbasis ersetzt werden kann ${ }^{14)}$.

\section{Der Spezialisierungskoeffizient}

Ein geeigneter Indikator zur Messung der regionalen Branchenstreuung ist der Spezialisierungskoeffizient. Er gibt an, wie stark die Branchenstreuung in einem Teilgebiet von derjenigen im Gesamtgebiet abweicht, d. h. mit seiner Hilfe lassen sich Aussagen über den Grad der strukturellen Einseitigkeit einer Region gewinnen. Der Spezialisierungskoeffizient wird - dargelegt an unserem Beispiel - folgendermaßen berechnet: 
1. Schritt: Berechnung des prozentualen Beschäftigtenanteils der einzelnen Industriebranchen an der Gesamtzahl der Industriebeschäftigten in einem Kanton.

2. Schritt: Dieselbe Berechnung wie im Schritt 1 für die Schweiz.

3. Schritt: Bildung der Differenzen zwischen den entsprechenden Anteilen.

4. Schritt: Addition des Betrages dieser Differenzen.

5. Schritt: Die Division dieser Summe durch 200 ergibt den Spezialisierungskoeffizienten.

$$
S_{j}=\frac{1}{200} \cdot \sum_{i=1}^{m}\left|\frac{b_{i j}}{\sum_{i=1}^{m} b_{i j}}-\frac{\sum_{j=1}^{n} b_{i j}}{\sum_{i=1}^{m} \sum_{j=1}^{n} b_{i j}}\right|
$$

$\mathrm{S}$ = Spezialisierungskoeffizient

$\mathrm{b}=$ Beschäftigte $\quad$ Beschäftigte der Branche $\mathrm{i}$

$=$ Branche $\quad b_{i j}=$ im Teilraum $j, d . h$. im vorliegenden

$\mathrm{j}=$ Teilraum $\quad \begin{aligned} & \text { Fall Beschäftigte in einer industriellen } \\ & \text { Wirtschaftsgruppe in einem Kanton }\end{aligned}$

$\mathrm{m}=$ Anzahl Branchen, d. h. im vorliegenden Fall neunzehn Wirtschaftsgruppen (20-38)

$\mathrm{n}$ = Anzahl Teilräume, d. h. im vorliegenden Fall fünfundzwanzig Kantone.

Ein Wert von $\mathrm{S}=0$ bedeutet, daß das Teilgebiet und das Gesamtgebiet identische Branchenstrukturen besitzen. Demgegenüber entspricht $S=1$ einer (theoretischen) Situation, bei welcher in einer Region nur eine einzige Branche vorkommt, welche in allen übrigen Teilgebieten nicht vorhanden ist.

Wie ISARD ${ }^{15)}$ und HOOVER ${ }^{16)}$ ausführen, kann der Spezialisierungskoeffizient für sehr verschiedene Untersuchungen verwendet werden, beispielsweise beim Vergleich der Altersstruktur der Bevölkerung in verschiedenen Regionen, der sprachlichen oder ethnischen Zusammensetzung der Bevölkerung usw. Der zeitliche Vergleich des Spezialisierungskoeffizienten zeigt auf, ob sich eine bestimmte Struktur in einem Teilgebiet im Laufe der Zeit von der (Durchschnitts)struktur des Gesamtgebietes entfernt oder sich ihr nähert.

\section{Der Konzentrationskoeffizient}

Der Konzentrationskoeffizient bildet gewissermaßen das Spiegelbild zum Spezialisierungskoeffizienten.
«Er zeigt die relative regionale Konzentration einer Teilgröße in bezug auf eine nationale Gesamtgröße über alle Regionen an ${ }^{17) . » ~ D i e ~ B e r e c h n u n g ~ e r f o l g t ~}$ sehr ähnlich wie beim Spezialisierungskoeffizienten:

1. Schritt: Berechnung des prozentualen Beschäftigtenanteils der einzelnen Kantone an der Gesamtzahl der Industriebeschäftigten in einer Industriebranche.

Schritt 2 bis 5: Analog der Berechnungen beim Spezialisierungskoeffizienten.

$$
K_{i}=\frac{1}{200} \cdot \sum_{j=1}^{n}\left|\frac{b_{i j}}{\sum_{j=1}^{n} b_{i j}}-\frac{\sum_{i=1}^{m} b_{i j}}{\sum_{i=1}^{m} \sum_{j=1}^{n} b_{i j}}\right|
$$

$\mathrm{K}=$ Konzentrationskoeffizient

Übrige Simbole gleich wie beim Spezialisierungskoeffizienten.

Je näher der Wert von $\mathrm{K}$ bei 0 liegt, desto gleichmäßiger ist die Branche über die Regionen verteilt. Je näher das Ergebnis bei 1 liegt, desto größer ist die relative regionale Konzentration der untersuchten Branche. Der Wert 1 ergibt sich, wenn die gesamte Branche in einer einzigen Region konzentriert ist. Gleichmäßigkeit bedeutet, daß die untersuchte Branche regional ebenso verteilt ist, wie die gesamte Industrie. Allgemein gilt, daß Konzentrationskoeffizienten mit zunehmender Größe der Regionen an Aussagekraft verlieren ${ }^{18)}$.

Die Konzentrationskoeffizienten sollten ursprünglich dazu dienen, eine Klassifizierung der verschiedenen Industriebranchen im Hinblick auf ihre Eignung für die Ansiedlung in Notstandsgebieten zu ermöglichen. Man ging dabei von der Annahme aus, $\mathrm{da} ß$ Industriebranchen, deren Standorte relativ breit gestreut sind (niedriger Konzentrationskoeffizient), keine bestimmten Anforderungen an ihren Standort stellen und daher ohne große wirtschaftliche Verluste verlagert werden können ${ }^{19)}$. Nach STORBECK ${ }^{20}$ ) kann aber diese Kennziffer nicht nur als Konzentrationsmaßstab verwendet werden, sondern kann auch zur Beschreibung der relativen Ausstattung der Regionen mit Einrichtungen der Infrastruktur und Nahversorgung eingesetzt werden.

Eng verwandt mit dem Konzentrationskoeffizienten ist der Koeffizient der räumlichen Verflechtung21). Diese Kennziffer wird in der Weise ermittelt, daß 
die prozentualen Anteile der in den einzelnen Teilräumen in einer Industriebranche Beschäftigten nicht auf die entsprechenden Anteile der Industriebeschäftigten insgesamt bezogen werden, sondern nacheinander auf die prozentualen Anteile der Beschäftigten in anderen Industriebranchen. Die Berechnungsformel lautet folgendermaßen:

$$
V_{h i}=\frac{1}{200} \cdot \sum_{j=1}^{n}\left|\frac{b_{i j}}{\sum_{j=1}^{n} b_{i j}}-\frac{b_{h j}}{\sum_{j=1}^{n} b_{h j}}\right|
$$

$\mathrm{V}=$ Koeffizient der räumlichen Verflechtung

$\mathrm{h}=$ Branche $\mathrm{h}$

$\mathrm{i}=$ Branche

Übrige Symbole gleich wie beim Spezialisierungskoeffizienten.

Mit Hilfe dieses Koeffizienten kann festgestellt werden, welche Industrien vorwiegend im räumlichen Verbund, d. h. jeweils in den gleichen Regionen lokalisiert sind. Daraus können unter Einbezug von weiteren Merkmalen Rückschlüsse auf deren $\mathrm{Ab}$ hängigkeiten von Agglomerationseffekten (localisation economies und urbanisation economies) gezogen werden (zz). Der Wert V $=0$ weist darauf hin, $\mathrm{da}$ zwei Industriebranchen stets im räumlichen Verbund auftreten, während ein Wert von 1 dann vorkommt, wenn die beiden Industriebranchen nie in der gleichen Region vertreten sind.

Von der rechnerischen Ableitung her bestehen auch enge Beziehungen zwischen dem Konzentrationssowie Spezialisierungskoeffizienten einerseits und dem Standortquotienten ${ }^{23)}$ andererseits:

$$
S Q_{i j}=\frac{\frac{b_{i j}}{\sum_{i=1}^{m} b_{i j}}}{\sum_{j=1}^{n} b_{i j}}=\frac{\frac{b_{i j}}{\sum_{j=1}^{n} b_{i j}}}{\sum_{i=1}^{m} \sum_{j=1}^{n} b_{i j} b_{i j}}
$$

$\mathrm{SQ}=$ Standortquotient

Übrige Symbole gleich wie beim Spezialisierungskoeffizienten.

Der Standortquotient gibt an, wie stark eine bestimmte Branche in einem Teilgebiet im Vergleich zum Gesamtgebiet über-, bzw. untervertreten ist. Bei einem Wert dieses Quotienten von 1 ist die Branche im Teilgebiet und im Gesamtgebiet relativ gleich stark vertreten. Werte über 1 bedeuten überdurchschnittliche, Werte unter 1 unterdurchschnittliche Repräsentation einer Branche im Teilgebiet ${ }^{24)}$.

\section{Resultate}

Die beiden folgenden Tabellen ${ }^{25}$ ) (Tab. 1 und Tab. 2) vermitteln einen Überblick über die mit den Daten der Industriestatistiken 1967 und 1972 errechneten Spezialisierungskoeffizienten für die Kantone und Konzentrationskoeffizienten für die Wirtschaftsgruppen 20-38. In den Tabellen werden die Kantone und Wirtschaftsgruppen geordnet nach der Größe ihres Spezialisierungs-, bzw. Konzentrationskoeffizienten für 1972 aufgeführt:

\begin{tabular}{|c|c|c|c|}
\hline Kanton & 1967 & 1972 & $\begin{array}{l}\text { Entwicklung } \\
1967 / 1972\end{array}$ \\
\hline Aarau & 0.132 & 0.127 & $\mathrm{D}$ \\
\hline Waadt & 0.177 & 0.149 & $\mathrm{D}$ \\
\hline Bern & 0.165 & 0.155 & $\mathrm{D}$ \\
\hline Luzern & 0.155 & 0.164 & $\mathrm{~S}$ \\
\hline Zürich & 0.190 & 0.205 & $\mathrm{~S}$ \\
\hline Thurgau & 0.215 & 0.205 & D \\
\hline St. Gallen & 0.231 & 0.208 & $\mathrm{D}$ \\
\hline Genf & 0.215 & 0.218 & $\mathrm{~S}$ \\
\hline Basel-Landschaft & 0.213 & 0.227 & $\mathrm{~S}$ \\
\hline Freiburg & 0.298 & 0.241 & $\mathrm{D}$ \\
\hline Schaffhausen & 0.259 & 0.271 & S \\
\hline Graubünden & 0.321 & 0.289 & $\mathrm{D}$ \\
\hline Schwyz & 0.334 & 0.296 & $\mathrm{D}$ \\
\hline Tessin & 0.301 & 0.304 & $\mathrm{~S}$ \\
\hline Solothurn & 0.349 & 0.319 & $\mathrm{D}$ \\
\hline Glarus & 0.403 & 0.353 & $\mathrm{D}$ \\
\hline Uri & 0.369 & 0.368 & $\mathrm{D}$ \\
\hline Zug & 0.396 & 0.386 & $\mathrm{D}$ \\
\hline Nidwalden & 0.419 & 0.430 & $\mathrm{~S}$ \\
\hline Wallis & 0.477 & 0.448 & $\mathrm{D}$ \\
\hline Obwalden & 0.527 & 0.453 & $\mathrm{D}$ \\
\hline Neuenburg & 0.464 & 0.480 & $\mathrm{~S}$ \\
\hline Appenzell-Außerrhoden & 0.507 & 0.485 & $\mathrm{D}$ \\
\hline Basel-Stadt & 0.514 & 0.582 & $\mathrm{~S}$ \\
\hline Appenzell-Innerrhoden & 0.639 & 0.618 & $\mathrm{D}$ \\
\hline $\mathrm{D}=$ Diversifikation & ezialisi & ung & \\
\hline
\end{tabular}

Tabelle 1: Spezialisierungskoeffizient 
Tabelle 2: Konzentrationskoeffizient

\begin{tabular}{llll}
\hline Wirtschaftsgruppe & 1967 & 1972 & Entwicklung 1967/1972 \\
\hline 34 Metallindustrie und -gewerbe & & & \\
35 Maschinen, Apparate, Fahrzeuge & 0.148 & 0.134 & $\mathrm{D}$ \\
20 Herstellung von Nahrungs- und Futtermitteln & 0.150 & 0.149 & $\mathrm{D}$ \\
25 Verarbeitung von Holz und Kork (ohne Zimmerei) & 0.164 & 0.169 & $\mathrm{~K}$ \\
28 Graphisches Gewerbe & 0.176 & 0.183 & $\mathrm{~K}$ \\
27 Papierindustrie & 0.191 & 0.189 & $\mathrm{D}$ \\
33 Bearbeitung von Steinen und Erden & 0.190 & 0.194 & $\mathrm{~K}$ \\
21 Herstellung von Spirituosen und Getränken & 0.221 & 0.216 & $\mathrm{D}$ \\
24 Herstellung von Kleidern, Wäsche und Schuhen; Bettwaren & 0.228 & 0.238 & $\mathrm{~K}$ \\
30 Kautschukindustrie, Kunststoffverarbeitung & 0.248 & 0.276 & $\mathrm{~K}$ \\
29 Herstellung und Bearbeitung von Leder (ohne Schuhe) & 0.324 & 0.305 & $\mathrm{D}$ \\
23 Textilindustrie & 0.314 & 0.308 & $\mathrm{D}$ \\
31 Chemische Industrie & 0.337 & 0.328 & $\mathrm{D}$ \\
26 Kinderwagen, Spielwaren, Sportgeräte & 0.466 & 0.480 & $\mathrm{~K}$ \\
38 Musikinstrumente & 0.391 & 0.484 & $\mathrm{~K}$ \\
22 Tabakindustrie & 0.462 & 0.519 & $\mathrm{~K}$ \\
37 Bijouterie, Gravier-, Prägeanstalten & 0.521 & 0.532 & $\mathrm{~K}$ \\
36 Uhrenindustrie & 0.501 & 0.548 & $\mathrm{~K}$ \\
32 Verarbeitung von Mineralöl & 0.568 & 0.569 & $\mathrm{D}$ \\
D = Dispersion K $\quad$ K Konzentration & 0.820 & 0.813 & $\mathrm{D}$ \\
\hline
\end{tabular}

Die Veränderung der kantonalen Spezialisierungskoeffizienten zwischen 1967 und 1972 zeigt, daß sich die Einseitigkeit der kantonalen Industriestrukturen weiterhin verringert hat. «Die Standorte der einzelnen Industrien waren früher stark nach Landesgegenden spezialisiert. Von den wichtigeren älteren Industrien war die Stickerei ausschließlich im Sankt-Gallisch-Appenzellischen verbreitet, die Baumwollspinnerei im Zürcher Oberland und in Glarus konzentriert, die Seidenstoffweberei vor allem auf dem linken Zürichseeufer, die Seidenbandindustrie in beiden Basel, die Uhrenindustrie im Jura angesiedelt. Mit der Zeit hat sich aber diese örtliche Spezialisierung und Differenzierung der Industrie abgeschliffen. Einige der historischen Industrien wie die Stickerei sind noch heute ganz auf ihre ursprünglichen Standorte beschränkt. Sie haben aber an Bedeutung verloren und geben daher dem Industriekörper nicht mehr im gleichen Maße das Gepräge wie früher. Diejenigen älteren Industrien, die ihre
Bedeutung bis heute zu behaupten vermochten, sind zum mindesten sporadisch über ihre ursprünglichen Gebiete hinausgedrungen. Vor allem aber haben sich die neueren Industrien in der Regel von vornherein stark zerstreut. Schon die Maschinen- und die Metallindustrie haben sich über das ganze Mittelland hin entwickelt, und das gleiche gilt für die verschiedenen Zweige der Nahrungs- und Genußmittelindustrie. Die chemische Industrie, die sich zur Hauptsache auf Basel konzentriert, bildet in dieser Hinsicht unter den neueren Industrien eine Ausnahme. Das Gesagte soll nicht heißen, daß überhaupt keine Spezialisierung der einzelnen Industriezweige nach Landesgegenden mehr besteht, aber die ausgesprochen monoindustriellen Gebiete sind seltener geworden26).» Betrachtet man eine Industriekarte27) oder eine Tabelle mit den Standortquotienten ${ }^{28)}$, so kann man doch immer noch folgende charakteristische regionale Branchenverteilung in der Schweiz feststellen: 
- Textilindustrie

- Bekleidungsindustrie

- Holzverarbeitung

- Chemische Industrie

- Uhrenindustrie
Ostschweiz

Ostschweiz und Tessin

Innerschweiz

Nordwestschweiz

Jura
Die regionalen Branchenschwerpunkte haben sich aber auch deshalb entschärft, weil heute größere Unternehmungen immer mehr auf eine sortiments- und absatzmäßige Diversifikation achten. Ferner ist zu berücksichtigen, daß die statistischen Branchenbegriffe im Laufe der jüngsten Entwicklung an Aussagekraft verloren haben ${ }^{29)}$.

Von 1967 bis 1972 hat sich der durchschnittliche kantonale Spezialisierungskoeffizient von 0.331 auf 0.319 verringert. Irgendwelche statistisch gesicherte Beziehungen zwischen der Veränderung der kantonalen Industriebeschäftigtenzahlen und der $\mathrm{Zu}$ - oder Abnahme der Spezialisierungskoeffizienten ließen sich für die untersuchte Zeitperiode von fünf Jahren nicht feststellen. Das bedeutet, daß auf Grund des vorliegenden Untersuchungsmaterials keine Aussage darüber gemacht werden kann, ob eine Industriebeschäftigtenzunahme in einem Kanton eher zu einer Spezialisierung oder zu einer Diversifikation der betreffenden kantonalen Industriestruktur führt. Entsprechendes gilt auch bei einer Abnahme der Industriebeschäftigten.

Betrachtet man die Entwicklung der Konzentrationskoeffizienten in den letzten fünf Jahren, so erkennt man, daß die Entwicklung ziemlich uneinheitlich war. Die Hälfte der Branchen verzeichnete eine räumliche Konzentration, die andere Hälfte eine Dispersion. Dieses Bild ändert sich allerdings, wenn man die Entwicklung der Konzentrationskoeffizienten mit der Beschäftigtenentwicklung in den einzelnen Industriebranchen vergleicht. Zwischen diesen beiden Größen ist folgender zu 95\% gesicherter statistischer Zusammenhang ${ }^{30}$ ) festzustellen: Eine Beschäftigtenabnahme in einer Industriebranche führt in der Regel zu einer Zunahme des Konzentrationskoeffizienten, bzw. ein Beschäftigtenzuwachs zu einer Verringerung des Konzentrationskoeffizienten.

FLORENCE 31) hat in Großbritannien und schwächer in den USA feststellen können, daß in den Industrien mit der relativ stärksten räumlichen Konzentration der Standorte Mittelbetriebe vorherrschen.
Mit Hilfe unseres Untersuchungsmaterials konnte eine Beziehung zwischen Betriebsgröße und Konzentrationskoeffizient für die Schweiz nicht nachgewiesen werden.

Sofern die bereits erwähnte Annahme stimmt, daß Industriebranchen mit einem niedrigen Konzentrationskoeffizienten keine bestimmten Anforderungen an ihren Standort stellen und deshalb besonders leicht verlagert werden können, würde das bedeuten, daß sich in der Schweiz am ehesten Betriebe der Metall- und Maschinenindustrie für Verlagerungen eignen würden. Das heißt, daß man bei der Suche nach geeigneten Industriebetrieben für die Ansiedlung in "unterentwickelten» Regionen der Schweiz diesen beiden Wirtschaftsgruppen besondere Aufmerksamkeit schenken sollte ${ }^{32}$ ).

Bewußt wird darauf verzichtet, die Berechnungsresultate mit Hilfe von Spezialisierungs- und Konzentrationskurven graphisch darzustellen ${ }^{33)}$. Die Interpretation solcher Schaubilder erfordert höchste Vorsicht. Bei der Spezialisierungskurve beispielsweise geht es lediglich darum, die strukturelle Einseitigkeit bzw. Ausgewogenheit einer Region zu veranschaulichen. Besondere Schwierigkeiten ergeben sich beim Vergleich von Spezialisierungskurven verschiedener Teilgebiete, bzw. beim Vergleich von Konzentrationskurven verschiedener Branchen. Dazu kommt, daß die "Gleichheitslinien» bei diesen Darstellungen - im Gegensatz zur Deutung der Gleichheitslinie in der Lorenz-Kurve - lediglich den optischen Vergleich erleichtern ${ }^{34)}$.

\section{Regionalpolitische Folgerungen 35 )}

«Das Ziel einer die Branchenstruktur anvisierenden staatlichen Strukturpolitik läßt sich wohl so formulieren, daß bei einem branchenspezifischen Beschäftigungsrückschlag jeder arbeitslos gewordene Arbeitnehmer mit hoher Wahrscheinlichkeit ohne Wohnortswechsel einen neuen Arbeitsplatz finden sollte ${ }^{36)}$.» Dabei darf man sich allerdings nicht nur auf die industrielle Branchenstruktur abstützen. An dieser Stelle soll lediglich auf diejenigen Regionen hingewiesen werden, welche sehr stark vom Fremdenverkehr abhängig sind. Dieses Ziel der Konjunkturresistenz und Beschäftigungsstabilität läßt zum mindesten für bestimmte Regionen und Kantone 
eine gegenüber dem Ist-Zustand ausgeglichenere, homogenere Branchenstruktur als wünschenswert erscheinen. Im industriellen Bereich dürfte dies vor allem für die Kantone Appenzell, Basel-Stadt, Neuenburg, Unterwalden und Wallis gelten.

Zur Verbesserung unbefriedigender regionaler Branchenverteilungen sind folgende Maßnahmen denkbar:

- Erhöhung der Transparenz, z. B. auf dem Arbeitsmarkt

- Schaffung von regionalen, bzw. kantonalen Entwicklungsimpulsen

- Verbesserung der regionalen, bzw. kantonalen Standortgunst ${ }^{37)}$.

Die regional ungleichmäßige Verteilung der Standortfaktoren darf, neben wirtschaftshistorischen Gegebenheiten, wohl als wichtigste Ursache eines unbefriedigenden Homogenitätsgrades der Branchenstruktur bezeichnet werden. Auf der folgenden Tabelle (Tab. 3) wird angegeben, für welche Industriebranchen in den einzelnen Kantonen die Standortvoraussetzungen verbessert werden müßten, um eine homogenere kantonale Industriestruktur zu erreichen. Dabei werden nur die drei Wachstumsindustrien Chemische Industrie, Metallindustrie und Maschinenindustrie berücksichtigt, da für diese drei Branchen das größte absolute Wachstum prognostiziert wird ${ }^{38}$ ).

Dies zeigt sehr deutlich, was bereits früher, bei der Untersuchung der Regional-, Standort- und Strukturfaktoren festgestellt worden ist ${ }^{39)}$, daß bei kantonalen Bemühungen zur Industrieansiedlung ${ }^{40)}$ der branchenmäßigen Zusammensetzung der Industrie große Beachtung geschenkt werden muß. «Man sollte nicht einfach jede erreichbare Ansiedlung akzeptieren, sondern eine genaue Analyse vornehmen, was der Region langfristig nützen kann ${ }^{41}$ ).»

$\mathrm{Ob}$, und wenn ja, wie weit die öffentliche Hand die industriellen Standortvoraussetzungen in einer Region verbessern soll ${ }^{42}$ ), um den Homogenitätsgrad zu erhöhen, hängt davon $a b$, welche politischen Vorstellungen über die optimale Industriestruktur bestehen. Diese sind wiederum davon abhängig, welche Gewichte dem Wirtschafts-, bzw. Industriewachstum einerseits und der Beschäftigungsstabilität, d. h. dem Arbeitsplatzwechsel ohne Wohnortswechsel, anderseits beigemessen werden. «Aus wis- senschaftlicher Sicht läßt sich zur Fixierung dieses Optimums wenig beitragen, da es sich hierbei um politische Wertungen handelt ${ }^{43)}$.»

Die vorliegende Untersuchung hofft einen kleinen Beitrag zu einer sachlich geführten Diskussion über Probleme der Regionalstruktur, insbesondere der kantonalen Industriestrukturen, leisten zu können.

Tabelle 3:

Liste der besonders zu fördernden Industriebranchen

\begin{tabular}{ll}
\hline Kanton & Industriebranchen \\
\hline Zürich & Chemie, Metall \\
Bern & Chemie \\
Luzern & Chemie \\
Uri & Chemie \\
Schwyz & Chemie, Maschinen \\
Obwalden & Chemie, Metall, Maschinen \\
Nidwalden & Chemie, Metall \\
Glarus & Chemie, Metall \\
Zug & Chemie, Metall \\
Freiburg & Metall, Maschinen \\
Solothurn & Chemie, Maschinen \\
Basel-Stadt & Metall, Maschinen \\
Basel-Landschaft & Maschinen \\
Schaffhausen & Chemie \\
Appenzell-Außerrh. & Chemie, Maschinen \\
Appenzell-Innerrh. & Chemie, Maschinen \\
St. Gallen & Chemie \\
Graubünden & Metall, Maschinen \\
Aargau & Chemie \\
Thurgau & Chemie \\
Tessin & Chemie, Maschinen \\
Waadt & Chemie \\
Wallis & Maschinen \\
Neuenburg & Chemie, Metall, Maschinen \\
Genf & Metall \\
\hline
\end{tabular}

Bei der Zusammenstellung dieser Liste wurden u. a. die kantonalen Standortquotienten der drei Industriebranchen im Jahre 1972 berücksichtigt. 


\section{Zusammenfassung}

Die Regionalforschung hat verschiedene methodische Hilfsmittel entwickelt, mit denen m. E. auch im Rahmen von geographischen Untersuchungen vermehrt gearbeitet werden sollte. Um diese Instrumente etwas bekannter zu machen, werden zwei davon, nämlich der Spezialisierungskoeffizient und der Konzentrationskoeffizient, an einem schweizerischen Beispiel dargestellt. Die Analyse der schweizerischen Industriestruktur mit Hilfe dieser beiden Kennziffern zeigt deutlich einerseits die unterschiedliche Homogenität und Heterogenität der kantonalen Branchenstrukturen sowie anderseits die räumliche Konzentration und Dispersion der verschiedenen industriellen Wirtschaftsgruppen. Die Frage, wie weit nun von der heutigen, realen Branchenstruktur in Richtung einer bezüglich Beschäftigungsstabilität und Wirtschafts-, bzw. Industriewachstum optimalen Branchenstruktur abgewichen werden soll, vermag die vorliegende Untersuchung nicht zu beantworten. Ein solcher Entscheid läßt sich nicht auf wissenschaftlicher, sondern nur auf politischer Ebene treffen. Die Wissenschaft und damit auch die Geographie kann aber dazu Entscheidungsunterlagen liefern.

\section{Anmerkungen und Literaturverzeichnis}

1) J. H. MÜLLER, Neuere Methoden der Regionalanalyse und ihre Anwendbarkeit auf kleinere Räume, in H. K. SCHNEIDER, (Hrsg), Beiträge zur Regionalpolitik, Schriften des Vereins für Sozialpolitik, N.F., Bd. 41, Berlin 1968, S. 90.

2) J. H. MULLER, Methoden zur regionalen Analyse und Prognose, Taschenbücher zur Raumplanung, Bd. 1, Hannover 1973, S. 44.

3) Vgl. dazu z. B. W. ISARD, Methods of Regional Analysis, An Introduction to Regional Science, M.I.T. Press, 2nd edition, Cambridge (Mass.) 1962, S. $232 \mathrm{ff}$.

4) G. FISCHER, Praxisorientierte Theorie der Regionalforschung, Tübingen 1973, S. 95.

5) Vgl. dazu J.H.MÚLLER, Neuere Methoden ... a. a. O., S. 90.
6) Nach D. STORBECK, Zur Methodik und Problematik von Maßstäben der regionalen Konzentration, in Raumforschung und Raumordnung, Heft 5/6, 1969 , S. 214 ff.

7) Vgl. dazu z. B. E. M. HOOVER, An Introduction to Regional Economics, New York 1971, S. $213 \mathrm{ff}$.

8) Als Beispiele von geographischen Untersuchungen, in welchen solche Kennziffern verwendet werden, seien erwähnt: H. ELSASSER, Veränderungen der kantonalen Beschäftigtenstrukturen in Industrie und Handwerk in der Schweiz, 1955-1965, in Geographica Helvetica Nr. 4, 1972, S. 148 ff. und $\mathrm{H}$. ELSASSER, die neue Karte «Industrie und Gewerbe, Übersicht» des Atlas der Schweiz, in Geographica Helvetica Nr. 4, 1972, S. 158 ff.

9) Die Ergebnisse der Eidgenössischen Industriestatistik vom September 1967 wurden veröffentlicht in Die Volkswirtschaft, Heft 7 und 8, Juli und August 1968, S. $405 \mathrm{ff}$. und $426 \mathrm{ff}$., diejenigen vom September 1972 im Heft 3, März 1973, S. 139 ff.

10) Bundesgesetz über die Raumplanung, Art. 79,

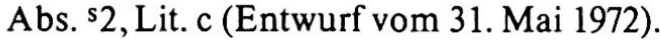

11) Arbeitsgruppe des Bundes für die Raumplanung, Raumplanung Schweiz. Hauptbericht, o. O., 1970, S. 78.

12) Prognos AG, Wirtschaftliche Entwicklungsmöglichkeiten des Landes Niedersachsen bis zum Jahre 1980, Basel 1967, S. 27.

13) W. BICKEL, Die Volkswirtschaft der Schweiz, Entwicklung und Struktur, Aarau und Frankfurt a. M., 1973, S.209.

14) Nach D.STORBECK, Zur Methodik ... a. a. O., S. 219.

15) W. ISARD, Methods ... a. a. O., S. 271.

16) E. HOOVER, An Introduction ... a. a. O., S. $212 \mathrm{f}$.

17) D. STORBECK, Zur Methodik ... a. a. O., S. 218.

18) Vgl. dazu E. LaUSCHMANN, Grundlagen einer Theorie der Regionalpolitik, Taschenbücher zur Raumplanung, Bd. 2, Hannover 1973, S. 132.

19) Nach E. LauschmanN, Grundlagen ... a. a. O., S. 130

20) D. STORBECK, Zur Methodik ... a. a. O., S. 219.

21) Vgl. dazu E. LausChMANN, Grundlagen ... a. a. O., S. 134 , D. M. SMITH, Industrial Location, An Eco- 
nomic Geographical Analysis, New York, London u. a. 1971, S. 394 f. und M. STREIT, Über die Bedeutung des räumlichen Verbunds im Bereich der Industrie, Schriftenreihe Annales Universitatis Saraviensis, Rechts- und wirtschaftswissenschaftliche Abteilung, Heft 27, Köln, Berlin u. a., S. 46 f.

22) Vgl. dazu R. ABT, Agglomerationseffekte in der schweizerischen Industrie, ORL-Schriftenreihe Nr. 17, ORL-Institut ETH Zürich, 1973.

23) Vgl. dazu z. B. H. ElSASSER, Die neue Karte... a. a. O., S. 159.

24) Zur Anwendung des Standortquotient vgl. z. B. Y. K. ALAGH, u. a., Interregional Industrial Structure in a Developing Economy: a Conceptual Frame with a Case Study, in Journal of Regional Science, Vol. 11, No. 3, 1971, S. $301 \mathrm{ff}$.

25) Berechnungen ausgeführt von Herrn KURT ALTERMATT, ORL-Institut ETH, Zürich.

26) W. BICKEL, Die Volkswirtschaft ... a. a. O., S. $208 \mathrm{f}$.

27) Vgl. dazu die Tafeln 60-63 des Atlas' der Schweiz, Industrie und Gewerbe I-IV, Wabern-Bern 1972.

28) Eine solche Tabelle, berechnet mit den Daten der Eidgenössischen Betriebszählung 1965 findet sich im Artikel von H. ELSASSER, Die neue Karte... a. a. O., S. 160.

29) Nach Studienkommission für Preis-, Kosten- und Strukturfragen, Studien zur Regionalpolitik, Bern 1972.

30) Spearmanscher Rangkorrelationskoeffizient $\mathrm{R}=$ -0.398 .

31) Zitiert nach E. LAUSCHMANN, Grundlagen ... a. a. O., S. 133.

32) Zum Problem der wirtschaftlichen Förderung «unterentwickelter» Regionen in der Schweiz vgl. ins- besondere Arbeitsgruppe STOCKER, Grundlagen zu den Leitlinien für die Berggebietsförderung, EVD Bern 1972.

33) Vgl. dazu w. ISARD, Methods ... a. a. O., S. $273 \mathrm{ff}$.

34) Nach J. H. MÜlLER, Neuere Methoden ... a. a. O., S. 49.

35) Die nachfolgenden Ausführungen stützen sich im wesentlichen auf Studienkommissionen für Preis-, Kosten- und Strukturfragen, Studien zur Regionalpolitik, a. a. O.

36) Studienkommission, Studien zur Regionalpolitik, a. a. O., S 187.

37) Zum Problem der regionalen industriellen Standortgunst vgl. H. ELSASSER, Die industriellen Standortvoraussetzungen in der Schweiz, ORL-Arbeitsbericht Nr. 15, ORL-Institut ETH, Zürich 1970.

38) Nach Arbeitsgruppe Perspektivstudien, Entwicklungsperspektiven der schweizerischen Volkswirtschaft bis zum Jahre 2000, Teil III Branchenmäßige Entwicklungsperspektiven, Band I, St. Gallen 1971, S. 52, Tab. 4.

39) H. ELSASSER, Veränderungen... a. a. O., S. $148 \mathrm{ff}$.

40) Vgl. dazu H. ELSASSER, Maßnahmen zur Förderung der Industrieansiedlung in den Kantonen, in ORL-DISP Nr. 28 (1973), S. 14 ff.

41) H. GERFIN, Gesamtwirtschaftliches Wachstum und regionale Entwicklung, in Kyklos, Vol. XVII, (1964), S. 590.

42) Vgl. dazu H. R. MOCK und J. KUNDT, Steuerungsmöglichkeiten der industriellen Standortwahl, ORL-Arbeitsbericht Nr. 12, ORL-Institut ETH,Zürich 1970.

43) Studienkommission, Studien zur Regionalpolitik, a. a. O., S. 211. 